Sasha Kurlenkova

\title{
WHO SPEAKS WITH MY SYNTHESIZED VOICE? SOME ROLES OF OTHER-INITIATED REPAIR IN AUGMENTED INTARACTION
}

As conversation analysis shows, all talk is highly collaborative, and meaning is created dialogically and sequentially, via the concerted actions of all the participants involved. In the case of people with communication impairments, the collaborative character of talk is even more manifest. A speaker with dysarthria, for example, may communicate through typing their messages to a text-to-speech communication app or device, using a communication (alphabet) board, or gazing at objects. This article focuses on one type of co-construction effort aimed at helping an augmented speaker to communicate, a process that can be called other-initiated repair. Although this practice is a common way of achieving understanding with people who have communication needs, in some cases repair initiation is used to do more than that. In this paper, I conduct conversation analysis of a video-recording of naturalistic interactions inside a Russian-speaking family involving a 10-year-old girl with dysarthria who communicates with her parents through an eyetracker-controlled computer interface. In this case, her parents use the structural position of repair initiation on the girl's words not only to clarify the meaning of her message but to continue the preceding polemics over the mom's birthday present. I argue that although this is just one instance of the use of other-repair in playful communication between family members, the potentiality of providing the type of guessing which aligns with the guesser's interests is present in other repair sequences. This can be consequential for lives of people with communication needs when done in more official settings. Studying similar repair sequences can help better delineate 'good' scaffolding strategies in co-construction of speech of someone with communication needs.

Sasha Kurlenkova-PhD student at the Department of Media, Culture, and Communication, New York University, New York, USA. Email: ask689@nyu.edu 
Key words: atypical interactions, alternative and augmented communication, conversation analysis, other-initiated repair, agency, scaffolding, co-construction

DOI: 10.17323/727-0634-2021-19-4-585-600

The question of agency in the production of one's talk is probably one of the central issues when it comes to individuals with speech disabilities. A common problem is that others - parents, hospital staff, etc. - often get to speak on behalf of children and adults with communication impairments (Robillard 1999; Sequenzia, Grace 2017; Alper 2017). As Ivan Bakaidov, a Russian programmer with cerebral palsy, points out in a public talk: 'I happened to participate in events for people with disabilities, which looked like a club of talking moms and children quietly sitting next to them' (Bakaidov 2019). Chances are high that when their communicative abilities are impaired, their everyday choices and even life-changing decisions will be made without their direct involvement.

This concern, however, collides with another important aspect of the talk of individuals with speech disabilities - that for effective communication, they need a lot of skilful collaboration on the part of their interlocutors. And not only challenged speakers are in this position. As conversation analysis (CA) has shown, all talk is highly collaborative, and meaning is created dialogically and sequentially, via the concerted actions of all the participants involved, rather than through individual actions of an idealized autonomous speaker (Goodwin 2004; Auer et al. 2020). In the case of people with communication needs $(\mathrm{PwCN})$, this collaborative character of talk is even more manifest. The co-construction of talk can happen through the use of objects in the environment and the setting per se, through repair practices (described below), or the use of sequentiality of talk (e.g. tying one's talk or gestures to the talk of others). Hence, a speaker with dysarthria (a physical impairment of a person's motor speech apparatus which results into 'phonetic distortion' of their speech) may communicate through typing their messages to a text-to-speech communication app or device, using a communication (alphabet) board, or gazing/ pointing at objects. All of these require the cooperative actions of their interlocutors: such as reading from the screen, following one's pointing gestures on the board, or guessing what their gazes/vocalizations/typed messages mean.

An important part of the job of conversational partners of PwCN is to provide the right type of collaborative efforts (scaffolding) and at the same time not to take 'too much space' - to avoid attributing meanings that the speaker did not want to convey. Consider one anecdote from the author's ethnographic work with Ivan Bakaidov cited above. Our face-to-face interactions with Ivan had the following structure: each time Ivan would say something with his regular voice affected by dysarthria, I would repeat it as I got it, and in the next turn he would either confirm or reject my understanding. In one funny and, luckily, not very consequential situation, Ivan was talking about 
music he was recently listening to, and I caught myself hearing and suggesting a list of different food and drinks as candidates for his words. It turned out I was hungry and heard what was on my mind at that moment!

Similar types of misattributions during the guessing activities provided by conversational partners of PwCN is something I will explore in this article. I will analyse one sequence where parents of a girl with dysarthria who uses eyetracker-mediated computer program got involved in a rather long guessing game over the meaning of the girl's words. I will show what gets done in each of the guessing turns (or other-initiations of repair, in CA terminology) beyond achieving understanding, and what these actions say about how agency/authorship can be distributed between speakers and technologies in a complex ecology of augmented interaction.

\section{Augmented interaction: speaking through eyetracker and speech synthesizer}

One of the tools of Augmentative and Alternative Communication (AAC) which has recently risen in popularity are eyetrackers. It has already become essential for some PwCN who have dysarthria or anarthria, as well as no control over their arms and hands, but who retain control over their eye movements. The same way that many users would deploy their hands, a keyboard and a mouse to browse webpages or work on their computer, an augmented speaker may utilize their eyes and an eyetracker to input commands to the interface. It allows them to type their messages into a communication app with single letters on a virtual keyboard, or graphic-based cards.

This communication system is a hybrid interaction medium (FulcherRood, Higginbotham 2019: 373), since an augmented speaker first enters their talk with their eyes as a text or graphic, and then, using the 'sounding' function of a speech synthesizer, plays it out loud with a computer voice. Following Erving Goffman's classification of speakers (1979) as authors (creators of the words that are heard), animators (speakers who animate the words of someone else, such as students reciting a classical poem or re-enacting a story happened to them in the past), and principals (those accountable for what is said), we can say that the roles of author/principal and animator in the case of augmented speakers are divided between the person who types messages, and the machine who voices them out (Auer et al. 2020:379).

Despite its revolutionarily enabling function, speaking through eyetracker and speech synthesizer is fraught with some challenges for the participants. It is relatively time-consuming compared to mouth speaking and may substantially increase the time of one's turn. Nor does it provide the speaker with typical 'quick means' of correcting the understanding of their interlocutor, e.g. such as regular self-repair practices. Typing with one's eyes requires augmented speakers to pre-plan their messages, with little possibilities to participate in the 
conversation as an emergent phenomenon (see, Engelke, Higginbotham 2013; Higginbotham, Wilkins 1999; Fulcher-Rood, Higginbotham 2019).

These peculiarities of augmented talk create the need for one's conversational partner to contextualize augmented talk, relate it to the topic discussed previously, or confirm one's understanding. Guessing is one of the common strategies used to support the speech of $\mathrm{PwCN}$ in aided interactions. As Arlene Kraat (1987) notes in her comprehensive review of various studies on AAC, the partner of augmented speaker may guess the remainder of a word, phrase or sentence; they also may try to establish understanding by asking for confirmation, summarizing the different utterances of the aided speaker, or seeking elaboration of different elements (through a series of yes/no, 'Wh' or forced choice questions). Guessing, providing interpretations/confirming understanding of an unclear utterance produced with a speech-synthesizer, is a form of repair in CA terminology, a set of collaborative practices used to ensure understanding between the speakers. Let's briefly overview the different practices of repair in the next section.

\section{Repair in human communication}

In CA, people's systematic ways of dealing with troubles are called repair practices which consist of a trouble source (repairable), repair initiations and repair solutions. Anything is subject to repair, that is people can experience troubles with all aspects of conversation: producing coherent speech, hearing or understanding, as well as with what Gail Jefferson (1972) calls interactional errors, i.e. failures of adapting one's talk appropriately to one's co-participant or to the type of social situation.

CA distinguishes between four types of repair depending on the person who initiates repair and the one who completes it: (1) self-initiated self-repair, (2) other-initiated self-repair, (3) self-initiated other-repair and (4) other-initiated other-repair. These types are asymmetrically encountered in data. In typical interactions, people who produce trouble sources more often both initiate and complete repair of their own speech. On the other hand, if other people initiate repair, they tend to then yield the floor to the speaker of the trouble-source; so other-initiated self-repair is encountered more often that other-initiated other-repair (Schegloff et al. 1977). In 'atypical' interactions, however, 'the organization of repair may not be as inclined towards self-correction as Emanuel Schegloff et al. (1977) originally proposed'; the talk of challenged speakers can be othercompleted and even other-corrected (Laakso 2020: 282; Korbut, this volume).

In general, repair sequences maintain the progressivity of the ongoing conversation: since when people decide to stop to make sure they are on the same page, they diverge from their main topic. That is why we may say that repairs are somewhat dispreferred in a conversation: after all, people talk to each other not to grapple with every pronounced utterance, but to do other 
things - request something, express gratitude, make small talk, etc. Often people do not correct their own talk or the talk of others and just proceed with their current topic (Jefferson 2007). However, in the case of interactions of people with dysarthria, intelligibility issues, which are a quality of both the speaker and the listener (Bloch, Barnes 2020: 3), often arise. Another problem pertaining mostly to the use of AAC is that of comprehensibility when one's interlocutor understands every word of the speech synthesizer, but cannot understand what the speaker is doing with these words, or what these words relate to in the prior talk (Bloch, Wilkinson 2004). In such cases, one's interlocutor would often initiate repair on the dysarthric speaker's utterance or synthesized talk - to make sure they are on the same page with regard to what was just said, and what relevant next action it makes.

A common practice for restoring understanding in such cases is that of other-initiated self-completed repair that is an insert sequence following a problematic turn of a dysarthric/augmented speaker. In this case, (1) their interlocutors initiate a repair on their words, displaying their understanding of what was just said; and (2) the dysarthric/augmented speaker confirms, rejects or finetunes the repaired element. This basic practice, which is ubiquitous in 'typical' interactions, gives a speaker with dysarthria a chance to act like a final author or validator of the right understanding of their message. Although the utterance may be co-constructed, people with dysarthria/augmented speakers display 'epistemic authority' (Laakso 2020: 281) over their speech; they have the last say in validating whether the co-participant understood them correctly. However, as was just mentioned, instances of other-completed repair occur in 'atypical' interactions too, when both elements of repair sequence (repair initiation and repair completion) are produced by a non-disabled individual. Another difference from 'typical' talk is that, while in such talk understanding is often restored after a single repair sequence (Kitzinger 2013:252), in conversations with PwCN, repair attempts on the same trouble source may repeat multiple times ('a repair loop'), or complex and cascading troubles may occur (Bloch, Barnes 2020; Bloch, Wilkinson 2013).

While repair practices are aimed at eliminating uncertainty and reaching understanding between interlocutors, they simultaneously may and often do accomplish other actions too (Kitzinger 2013:242). This way, other-initiated selfrepairs can serve the function of managing one's relationships or identities. Otherinitiations of repair, for example, may signal an upcoming dispreferred response: along with fixing the troubles of hearing, speaking or understanding, they can perform such actions as 'doubt, non-alignment, disagreement, challenge, rejection, etc.' (Schegloff 1997:505). A person can also initiate repair on their interlocutor's words to treat something just said as in some way inapposite. This way, other-initiated self-repairs are used, for example, to instill etiquette rules in children (Kitzinger 2013:254). Similarly, people may show 'ritualized disbelief' in the form of other-initiated self-repair to respond to statements designed to invoke 
surprise (Wilkinson, Kitzinger 2006). These are just some of many examples of uses of this repair practice in 'typical' talk. The case below shows how, in augmented talk, other-initiated repair practice is reserved for a set of actions related to a playful 'tug-of-war' game between parents of a girl with dysarthria.

\section{Data and Pre-Analysis}

Analysis of this case is a part of the author's PhD project at NYU, approved by the university's Institutional Review Board (protocol \#IRB-FY 2021-5717). The interaction below was recorded inside a Russian-speaking family now living in Europe ${ }^{1}$. The family was asked to record their daily interactions using a go-pro camera and a program for recording the screen over the interval of six months, at times of their own choosing.

Julia (JUL) is a 10-year-old girl who lives with her MUM; her stepfather, MAN (this is how JUL calls him); and her little brother, BRO. Names of the participants were changed for anonymity reasons. Julia uses an eye-tracker to speak, constructing her messages from a set of cards in English using the Optikey Symbol app. The selection of cards does not happen immediately when JUL looks at them; JUL's settings are such that it takes around $0.6 \mathrm{sec}$. of uninterrupted looking for a card to get chosen, and then around $1 \mathrm{sec}$. of looking at the 'Play' sign at the entry line for the message to get played. If the utterance was already played once, the second time it is sometimes played immediately when JUL hits 'Play' button; and sometimes the software gets glitchy and fails to play the message more than once, even if the typist keeps pressing 'Play.' JUL's typing process is reflected on a big computer screen which hangs above her on the wall, at the eye level of the adults. This makes JUL's messages and the process of their composition available to the parents who can see them on the screen, in addition to hearing utterances voiced by the speech-synthesizer.

The family speaks in a combination of Russian and English, and uses a multimodal communication system involving the parents' speech, JUL's facial expressions, vocalizations and her utterances pronounced by the communication app. Gaze plays an important role in many of JUL's interactions, not only because she types her messages with it, but because she can use it in other semiotic activities, like looking at the thing she talks about, or looking away from people if they do not get her message. The latter convention is something the family came up with for situations when they do not have access to the computer, or when they want to clarify the meaning of JUL's synthesized utterance. It is a binary system of signs: to say 'yes' Julia looks at the speaker; to say 'no' she looks away from them.

\footnotetext{
${ }^{1}$ Please see the full fragment in here: (1) go-pro plane: https://youtu.be/OkrkzXsr5V8; (2) screen recording: https://youtu.be/mNGFAR8P8-o. In this paper, due to the size limitations, I will present the transcript and analyze only the very last part of the sequence (a complex other-initiated repair practice).
} 
Due to the size limitations of this paper, I will briefly analyse the sequence preceding the repair situation as it sets the overall activity in which MUM, JUL, and MAN get involved, and then look in more detail at the repair practice itself, and the actions carried out through it. The analysed fragment shows the family's Monday morning: MUM is tidying up the living room; JUL is sitting in her chair in front of the computer screen; MAN is watching TV and feeding Julia's little brother in the other half of the room. Right before this fragment, JUL starts discussing a possible gift for MUM's upcoming birthday. The video starts at the point when MUM asks JUL what kind of gift she is talking about, and comes up close to her. She then bends over JUL's ear and asks her in a playful, whispering voice to tell MAN that MUM needs a new phone (gazing in MAN's direction). This way, MUM playfully requests JUL to act as animator of her words to MAN, to speak on her behalf, but presenting JUL as the author and principal of this utterance. MUM also whispers JUL a couple of precise formulations for this request, the last one being 'Man mum need new phone' which JUL then types almost precisely (with the exception of one word 'phone' which is only present as 'iPhone' among default app cards).

Once finished typing, JUL voices her message twice. However, because of the rather inexpressive voice tone of the speech, on the one hand, MAN's rather distant position and his involvement in another activity (news watching and babysitting), on the other, he produces no visible reaction to JUL's voiced utterance. Then MUM makes another kind of contribution to JUL's talk: she advises JUL to whistle (pick 'Attention' card on the screen), and after 3 whistles not soliciting any reaction from MAN, she says in a louder voice: '[Name of MAN], she is whistling at you!' MAN then produces an open-class repair initiation ('What?'), in response to which JUL activates voicing of her message again. After a gap, MAN tells BRO to wait and complains about having to attend to two children simultaneously, to which MUM replies with a made-up argument that she needs MAN to switch the language at JUL's synthesizer (which is typically MAN's responsibility in the family). Only after these collaborative efforts does MAN come to their half of the room and looks at the screen.

When arrived, MAN does the minimal job of reading the utterance from the screen and goes back to his seat (without attending to MUM's request 'to fix the language'). Going back, he produces a delayed second pair part consisting of a partial repeat, and a 'weak' disagreement: 'New iphone? I don't know, her behaviour is very bad really.' At the end of MAN's turn, JUL clears her previous utterance and starts typing another one; these actions take her more than 30 seconds during which MUM and MAN do a sort of 'tug-of-war' game, giving arguments for and against MUM's getting a new phone.

Right after MAN's turn, MUM repeats JUL's utterance ('Mum needs a new lil phone'). This action is not aimed at helping MAN understand JUL's utterance (MAN has just read it himself from the screen), but rather serves to share authorship and acknowledge MUM's partisanship in this conversation. 
MAN then addresses JUL suggesting that with the new phone MUM will spend more time on it and less time with JUL. MUM and MAN start arguing among themselves about MUM's time on the old vs. new phone, changing participation framework and talking without orientation to JUL. This may be considered the point of MAN's public acceptance of MUM as the principal of JUL's initial request.

\section{'MUM I'M SORRY' repair sequence}

During the ensuing $5.5 \mathrm{sec}$ silence, MUM is looking at the screen while JUL is finishing her utterance. Let's look in detail at JUL's next turn which is treated as problematic by MUM and MAN, and what each speaker is doing in the following other-initiated repair sequence.

For transcription conventions please see the Table 1 of Higginbotham et al., present volume. The transliteration system was designed by Bolden (2008: 135-136). The English translation of spoken turns is done in bold for readers' convenience.

$\begin{array}{lll}01 & \text { JUL: } & ((\text { looks at MUM })) \\ 02 & \text { MUM: } & ((\text { looks at JUL, then at screen })) \\ 03 & \text { JUL: } & ((\text { looks at screen })) \\ 04 & \text { MUM: } & ((\text { looks at JUL })) \\ 05 & \text { JUL: } & \text { MUM [I'M SORRY } \\ 06 & \text { MUM: } & \quad[((\text { looks at screen })) \text { Zachto::? } \\ & & \text { For what } \\ & & ((\text { looks at MUM, turns head to MAN })) \\ 07 & \text { JUL: } & (1.0) \\ 08 & & \text { Chto on gavarit glupa }[\text { sti? } \\ 09 & \text { MUM: } & \text { what he speak nonsense }\end{array}$

\section{That he speaks nonsense?}

10 JUL: $\quad[(($ looks at MUM, then at screen $))$

$11 \quad(0.3)$

12 MAN: .hh he he.he.he ne:: $\mathrm{t}$ ana paprobovala karochi/

$$
\text { no she tried in short }
$$

.hh he he.he.he no, she tried, you know, 
and she didn't manage, (.) and now she says sorry

14 MUM:

[Ty izvi you sorry

You are

15

njaishsja chte u tibja ni paluchilos'?

sorry that PRT you not manage

sorry because you didn't manage

16 MAN: [.hh ]

17 MUM: [jigo] [ugavarit'?

him persuade

to persuade him?

18 JUL: $\quad[($ looks away from MUM $))$

19 MUM: $\mathrm{Ne}[\mathrm{t}$

no

No.

20 MAN: $\quad[\mathrm{Ne}:: \mathrm{t}$.

no

No.

21

Once she finishes typing her message ('MUM I'M SORRY'), JUL looks several times at MUM, presenting her message as complete and selecting MUM as the next speaker. MUM reads her message on the screen (line 2) but waits for JUL to voice it with the speech synthesizer before initiating a repair: 'For what?' This category-specific interrogative indicates that MUM recognizes JUL's utterance as an apology, but does not understand what she is sorry for. That is why she delays production of the second pair part to first gain a correct understanding of JUL's phrase. In response to MUM's interrogative, JUL turns her head in MAN's direction and looks at him for at least 1 sec. Note that, although MUM asks an open question, rather than a yes/no interrogative, which is more apt for quickly clarifying answers from JUL, JUL provides the 'best possible' repair solution in this situation-gazing at MAN to show that the trouble source relates to something he said or did.

MUM does not treat this response as satisfactory, and produces another repair initiation, this time providing a candidate understanding confirmable with a 'yes' or 'no' answer: 'That he speaks nonsense?' (line 9). This question draws on JUL's turn (looking at MAN), but turns it into a rather partisan 
formulation, where 'nonsense' is used to diminish the significance of MAN's words. This way, MUM not only makes another attempt at repairing understanding of JUL's words, but continues the 'verbal duel' with MAN that preceded the repair sequence.

In line 10, JUL rejects MUM's candidate understanding: JUL looks at MUM for at least a second, but then shifts her gaze back to the screen. JUL's initial looking at MUM may be discussed in the view of 'preference for agreement' (Sacks 1987) in yes/no questions, even more so given that MUM caresses JUL's head at the time of asking her question. Agreeing to MUM's formulation would mean, however, that JUL not only accepts MUM's interpretation of JUL's words, but her qualification of MAN's words as 'nonsense.'

Although MAN cannot see JUL's answer given his position in the room, he laughs and joins the guessing activity in lines $12-13$. His candidate understanding reveals how he sees the situation (JUL tried, did not manage, and says sorry), and is also a reply to MUM's suggestion in line 9 which contained an element of criticism of his words ('nonsense'). MUM interrupts him and reformulates this interpretation in the form of a yes/no question confirmable by JUL's gaze sign system (lines 14-15). Note that she adds the word 'persuade' to MAN's formulation ('did not manage'), interpreting JUL's utterance as her being sorry for the lost cause of convincing MAN on MUM's behalf. Projecting the end of the turn, JUL abruptly moves her head away from MUM and exaggeratedly looks up (so that her eyes are in the farthest position from MUMmeaning 'no, no'). This gesture is read unambiguously by both MUM and MAN as 'No' (lines 19-20).

22 JUL: [((turns head back to screen))

23 MAN: [Izvinis',/ say sorry

\section{Say that you are sorry.}

Mama dolzhna izvinit'sja/

mum needs say sorry

\section{Mum needs to say sorry}

26 JUL: [((presses 'Play' button))

27 MAN: Vot chta ana xochit skazat',= PRT what she wants say

\section{That's what she wants to say.=}

28 MUM: =Ne:: $\mathrm{t} /($.) ana napisala aajm sorri, no she wrote I'm sorry 
$=$ No, (.) she wrote I'm sorry,

29

$[(\cdot)$

30 JUL: $\quad[(($ presses 'Play' $2 \mathrm{x}$ time $))$

31 MAN: Aa: ty? sori=/pachimu ty ssorri,/

PRT you sorry why you sorry

Ah, you are sorry?= Why are you sorry?

32 JUL: $\quad[(($ presses 'Play' $3 x$ time $))$

33 MAN: [(0.3) tibe- tibe zhal'? $\uparrow /$

you you sorry

(0.3) You- you regret?

34

(0.8) Chigo? tibe zhal' Julia $\downarrow$,/

what you sorry Julia

(0.8) What do you regret, Julia?

35

(.)

36 MUM: Chto on ni pavëlsja?/

that he not buy in

That he didn't buy into it?

37

38 JUL: $\quad[(($ presses 'Play' $4 x$ time $)$

39 ((shifts gaze from screen to MUM, then away))

40 MUM: $\quad \mathrm{Ne}:: \downarrow$,

no

No

41 JUL: ((looks at screen))

In line 22, MAN gives a completely new candidate understanding of JUL's words, suggesting that it is MUM who needs to say sorry. Note that this phrase, as well as the previous repair initiation from MAN, is designed as an affirmative utterance, rather than a yes/no question to be confirmed or rejected by JUL. Given the preceding polemics between MUM and MAN, his utterance can be read in a way that he proffers his own desired version of the situation (MUM made an inadequate request of MAN, or conspired against him with JUL, so she needs to say sorry) in the form a repair initiation on JUL's utterance. Line 27 is added to counter this attributional danger: MAN highlights that this is what JUL wants to say, rather than MAN himself. Right after MAN's turn in line 24, JUL starts pressing the 'Play' button on the app to 
repeat her message. This way JUL tries to counter MAN's candidate understanding using the resources of an already typed utterance. However, the speech synthesizer is glitchy at this moment, and no sound follows.

MUM looks at JUL's face in line 25 (ignoring the screen) and not registering any response from her, rejects MAN's suggestion appealing to the grammatical structure of JUL's sentence. Note that here MUM and MAN do not wait as usual for JUL's repair completion, they negotiate the appropriateness of MAN's understanding between themselves. MUM completes repair acting as a 'representative' of JUL (prefacing her words with 'she said'), while JUL is busy doing something on the computer. At the same time, MUM's own position in the polemics with MAN gets advanced through rejecting MAN's suggestion (i.e. she is right, she has nothing to say sorry for).

In line 30, JUL makes a second attempt to voice her initial utterance, and it fails again. Meanwhile, MAN produces 'Ah,' a 'change-of-state token' (Heritage 1984), claiming the receipt of MUM's information as new. He then produces two repair initiations in line 31: first an open-class one, and then a category-specific why. Note that they are latched to each other, i.e. designed in a way not to give JUL an opportunity to respond to the $1^{\text {st }}$ question. Moreover, the form of the second repair initiation is such that JUL would not be able to give an answer to it, given her resources and time constraints in this already extended repair episode.

Again, right after MAN's utterance, JUL tries to activate the speech synthesizer which fails to work. At this point MUM throws a quick glance at the screen but either does not register JUL's attempt, or registers it but does not acknowledge it. Meanwhile, MAN continues his guessing activity, first producing the same open-class repair, but with the Russian word 'zhal" ('sorry, regret'), and then a category-specific interrogative slightly different from the previous one ('What do you regret, Julia?'). Notably, the second repair initiation, again, is not easily answerable by JUL since it is not done as a yes/no question.

At this point MUM again suggests a confirmable repair initiation: 'That he didn't buy into it?' This formulation is designed in a way to describe JUL's course of action as a (failed) joint plan with MUM to trick MAN into something. JUL makes the last attempt to voice her message (hence the delayed second pair part), then looks at MUM and shifts her gaze away from her. MUM voices her reply as 'No'. This round of repair is followed by two others. For brevity reasons, let's now jump to the ultimate part of this sequence when the repair is finally successfully completed.

$<$ Lines 42-54 were omitted $>$

55 MUM: Mam aim sorri no ju- e:: ty ni paluchish'

MUM I'm sorry but you PRT you not get

MUM, I'm sorry but you- You will not get 
56

a new phone?

57 JUL:

58

59 MUM:

60

个novyi [tilifon?/

new phone

a new phone?

((turns and looks at MAN))

Narmal'nyj blin ty vzjal eë padgavaril $\uparrow \mathrm{t}(\mathrm{h}) \mathrm{iper}$,

normal shoot you take her egg on now

Are you normal? You egged her on now!

In lines 55-56, MUM initiates repair by recycling the trouble source followed by 'but you will not get a new phone.' It presents JUL as being sorry about MUM's not getting a new phone, rather than for not managing to carry through MUM and JUL's interactional project. This statement is finally found satisfactory by JUL, who confirms it by abruptly looking at MUM in an overlap with the end of MUM's turn. JUL also smiles and nods as additional ways to show that she accepts this understanding. Notably, following the successful completion of repair activity, MUM does not reply to JUL (which would be a much delayed second pair part to JUL's initial problematic turn), but rather addresses MAN and in a jokingly accusing manner states that he egged JUL on to be on his side. This speaks to the fact that was what at stake, at least for MUM, in this insert sequence was not only finding the right understanding of JUL's words, but also continuing the polemics with MAN over the new phone.

\section{Conclusion}

Analysis of this sequence reveals the curious conundrum with which conversational partners of PwCN are faced - that of walking a fine line between providing sufficient opportunities for augmented communicators to speak for themselves, and that of co-construction, the ample collaborative work needed to support their talk. While in many face-to-face encounters it is the speech of PwCN which requires scaffolding efforts on the part of their conversational partners, what is peculiar here is that JUL at first speaks for MUM, acting as if she is the sole author and principal of MUM's request to MAN. (It is not only MUM's project too, since JUL is the one who raised the topic of MUM's birthday gift and then agreed to collaborate.) However, MUM is not fully exempt from the co-construction work: using her voice she secures the receipt of JUL's message, compensating for imperfections of the speech synthesizer's tone and distant location of the addressee. MUM puts considerable effort into this part of the job: she gives JUL advice on how to attract MAN's attention, uses her 
voice (whose dynamic qualities suit this task much better), and even 'lures' MAN into answering with a trick ('switch the language'). Later on, MUM also gets publicly recognized as the co-author (and co-principal for that matter) of JUL's utterance, when MUM and MAN switch to a conversation between themselves, without JUL's involvement.

When MAN rejects JUL's request, she initiates a turn to say, as we discover later, that she is sorry for MUM who will not get a new phone for her birthday. While each individual word of JUL's turn is clear to MUM and MAN, they experience troubles in understanding the overall meaning of it - issues with comprehensibility. The parents then take the role of JUL's collaborators in co-constructing the meaning of JUL's problematic utterance. They suggest a number of repair initiations and speak JUL's responsive actions out loud (Yes/No answers done with gaze).

However, apart from the action of resolving issues of understanding, JUL's parents continue their own 'tug-of-war game' using the structural position of repair initiations. MUM designs her candidate understandings in such a way as to diminish MAN's words or describe JUL's course of action as a part of the (failed) joint plan to persuade or 'trick' MAN into buying a phone. MAN suggests a candidate understanding which interprets JUL's utterance in such a way as to position MUM as the one who needs to apologize. This way, the repair sequence verges on the status of being the main one, rather than 'just' a side sequence it becomes a place for doing the same thing (parents' polemics) by other means.

Noteworthy, MAN formulates his repair initiations as either affirmative utterances, open-class or category-specific questions - none of which are quickly confirmable by JUL in the tight time constraints of this interactional setting. MUM, instead, acts as JUL's 'closest collaborator,' monitoring her embodied behaviour and formulating repair initiations in the form of yes/no questions confirmable with JUL's binary gaze signs. These differences may be caused by the different physical proximity of both parents to JUL, or a different understanding of their collaborative roles in the present interactional setting, among other reasons.

Although this is just one instance of the use of other-repair in playful communication between family members, I suggest that the potentiality of providing the type of guessing which aligns with the guesser's interests is present in other repair sequences. If done in more formal settings, such as a hospital, a courtroom, a social security office, etc., this type of co-construction can be very consequential for PwCN: the line between scaffolding and speaking for someone with communication needs may be crossed. This is complicated by the fact that sometimes gestures and gaze of someone with cerebral palsy or another neurological condition can be ambiguously read, while the recipients, especially unfamiliar ones, may not be sensitive or not pay attention to the cues given by such a speaker. Further analysis of similar other-initiated repair cases can show how misattributions are made and suggest ways of rebutting them for 
PwCN (with technologies and otherwise), as well as more accurate verification procedures for their conversational partners.

\section{Acknowledgements}

I am expressing my gratitude to people who shared their insightful comments about this case at EMCA_Ru data sessions and Atypical Interaction seminar.

\section{References}

Alper M. (2017) Giving Voice: Mobile Communication, Disability, and Inequality. Cambridge, MA: MIT Press.

Auer P., Bauer A., Hörmeyer I. (2020) How Can the 'Autonomous Speaker' Survive in Atypical Interaction? The Case of Anarthria and Aphasia. In: R. Wilkinson, J. Rae, G. Rasmussen (eds.) Atypical Interaction. The Impact of Communicative Impairments within Everyday Talk. Cham: Palgrave Macmillan:373-408.

Bakaidov I. (2019) Communication is the Basis of Human Life. TEDxSkoltech. Available at: https://www.youtube.com/watch? v=39cXt15_jA (accessed: 5.11.2021).

Bloch S., Wilkinson R. (2004) The Understandability of AAC: A Conversation Analysis Study of Acquired Dysarthria. AAC: Augmentative and Alternative Communication, 20 (4): $272-282$.

Bloch, S., Wilkinson, R. (2011) Acquired Dysarthria in Conversation: Methods of Resolving Understandability Problems. International Journal of Language and Communication Disorders, 46 (5): 510-523.

Bloch S., Wilkinson R. (2013) Multiple Troubles and Repair in Voice Output Communication Aid-mediated Interaction. In: N. Norén, Ch. Samuelsson, Ch. Plejert (eds.) Aided Communication in Everyday Interaction. Guilford: J\&R Press: 95-126.

Bloch S., Barnes S. (2020) Dysarthria and Other-initiated Repair in Everyday Conversation. Clinical Linguistics \& Phonetics, 34 (10-11): 1-21.

Bolden G. (2008) Reopening Russian Conversations: The Discourse Particle - to and the Negotiation of Interpersonal Accountability in Closings. Human Communication Research, 34 (1): 99-136.

Engelke Ch., Higginbotham D. J. (2013). Looking to Speak: On the Temporality of Misalignment in Interaction Involving an Augmented Communicator Using Eye-gaze Technology. Journal of Interactional Research in Communication Disorders, 4 (1): 95-122.

Fulcher-Rood K., Higginbotham J. (2019) Interacting with Persons Who Have ALS: Time, Media, Modality, and Collaboration via Speech-Generating Devices. Topics in Language Disorders, 39 (4): 370-388.

Goffman E. (1979) Footing. Semiotica, (25): 1-29.

Goodwin Ch. (2004) A Competent Speaker Who Can't Speak: The Social Life of Aphasia. Journal of Linguistic Anthropology, 14 (2): 151-170.

Jefferson G. (1972) Side Sequences. In: D. Sudnow (ed.) Studies in Social Interaction. New York: Free Press:294-338. 
Jefferson G. (2004) Glossary of Transcript Symbols with an Introduction. In: H. G. Lerner (ed.) Conversation Analysis: Studies From the First Generation. Amsterdam: John Benjamins: 13-31.

Jefferson G. (2007) Preliminary Notes on Abdicated Other-correction. Journal of Pragmatics, (39): 445-461.

Heritage J. C. (1984) A Change-of-State Token and Aspects of Its Sequential Placement. In: J. M. Atkinson, J. Heritage (eds.) Structures of Social Action. Cambridge: Cambridge University Press: 299-345.

Higginbotham D.J., Wilkins D.P. (1999) Slipping through the Timestream: Social Issues of Time and Timing in Augmented Interactions. In: D. Kovarsky, J.F. Duchan (eds.) Constructing (In)competence: Disabling Evaluations in Clinical and Social Interaction. Mahwah: Lawrence Erlbaum Associates, Inc.: 49-82.

Kitzinger C. (2013) Repair. In: J. Sidnell, T. Stivers (eds.) The Handbook of Conversation Analysis. Oxford: Blackwell: 229-256.

Kraat A. (1987) Communication Interaction between Aided and Natural Speakers: A State of the Art Report. Second Edition. Madison, WI: Trace Research and Development Center, University of Wisconsin-Madison.

Laakso M. (2020) Repair Organization in Linguistically Asymmetric Interaction: Comparing Child-Parent Conversations and Conversations Involving Speakers with Aphasia. In: R. Wilkinson, J. Rae, G. Rasmussen (eds.) Atypical Interaction. The Impact of Communicative Impairments within Everyday Talk. Cham: Palgrave Macmillan:257-287.

Norén N., Samuelsson C., Plejert C. (eds.) (2013) Aided Communication in Everyday Interaction. London: J\&R Press.

Oliver M. (1990) The Politics of Disablement. London: Palgrave Macmillan.

Robillard A. B. (1999). Meaning of a Disability: The Lived Experience of Paralysis. Philadelphia. Philadelphia: Temple University Press.

Sacks H. (1987) On the Preferences for Agreement and Contiguity in Sequences in Conversation, In: G. Button, J.R.E. Lee (eds.) Talk and Social Organisation. Clevedon: Multilingual Matters: 54-69.

Sequenzia A., Grace A. (2017) Typed Words Loud Voices. Fort Worth: Autonomous Press.

Sidnell J. (2010) Conversation Analysis: An Introduction. Malden: Wiley-Blackwell.

Schegloff E. A. (1997) Practices and Actions: Boundary Cases of Other-initiated Repair. Discourse Processes, 23 (3): 499-545.

Schegloff E. A., Jefferson G., Sacks H. (1977) The Preference for Self-correction in the Organization of Repair in Conversation. Language, 53 (2):361-382.

Wilkinson S., Kitzinger C. (2006) Surprise as an Interactional Achievement: Reaction Tokens in Conversation. Social Psychology Quarterly, 69 (2): 150-182. 